\title{
Observability of Coulomb-assisted quantum vacuum birefringence
}

\author{
N. Ahmadiniaz $\odot,{ }^{1}$ M. Bussmann, ${ }^{2,1}$ T. E. Cowan, ${ }^{1,3}$ A. Debus, ${ }^{1}$ T. Kluge, ${ }^{1}$ and R. Schützhold ${ }^{1,4}$ \\ ${ }^{1}$ Helmholtz-Zentrum Dresden-Rossendorf, Bautzner Landstraße 400, 01328 Dresden, Germany \\ ${ }^{2}$ Center for Advanced Systems Understanding (CASUS), 02826 Görlitz, Germany \\ ${ }^{3}$ Institut für Kern-und Teilchenphysik, Technische Universität Dresden, 01062 Dresden, Germany \\ ${ }^{4}$ Institut für Theoretische Physik, Technische Universität Dresden, 01062 Dresden, Germany
}

(Received 11 February 2021; accepted 3 June 2021; published 16 July 2021)

\begin{abstract}
We consider the scattering of an x-ray free-electron laser (XFEL) beam on the superposition of a strong magnetic field $\mathbf{B}_{\text {ext }}$ with the Coulomb field $\mathbf{E}_{\text {ext }}$ of a nucleus with charge number $Z$. In contrast to Delbrück scattering (Coulomb field only), the magnetic field $\mathbf{B}_{\text {ext }}$ introduces an asymmetry (i.e., polarization dependence) and renders the effective interaction volume quite large, while the nuclear Coulomb field facilitates a significant momentum transfer $\Delta \mathbf{k}$. For a field strength of $B_{\text {ext }}=10^{6} \mathrm{~T}$ (corresponding to an intensity of order $10^{22} \mathrm{~W} / \mathrm{cm}^{2}$ ) and an XFEL frequency of $24 \mathrm{keV}$, we find a differential cross section $d \sigma / d \Omega \sim 10^{-25} Z^{2} /(\Delta \mathbf{k})^{2}$ in forward direction for one nucleus. Thus, this effect might be observable in the near future at facilities such as the Helmholtz International Beamline for Extreme Fields at the European XFEL.
\end{abstract}

DOI: 10.1103/PhysRevD.104.L011902

\section{INTRODUCTION}

According to classical electrodynamics, electromagnetic waves in vacuum obey the superposition principle and thus do not influence each other. Quantum electrodynamics (QED), on the other hand, predicts that they do interact via their coupling to the fermionic degrees of freedom [1-3]. As a result, the quantum vacuum behaves as a polarizable medium, entailing phenomena such as refraction and birefringence.

The difficulties of observing these effects can be understood by recalling the characteristic scales of QED. First, the electron mass $m \approx 0.51 \mathrm{MeV} / c^{2}$ sets an energy scale where the associated length scale $\chi=\hbar /(m c) \approx 386 \mathrm{fm}$ is the Compton length. Second, the elementary charge $q$ can be used to construct the Schwinger critical field [4,5],

$$
E_{\text {crit }}=\frac{m^{2} c^{3}}{\hbar q} \approx 1.3 \times 10^{18} \frac{\mathrm{V}}{\mathrm{m}}
$$

where the corresponding magnetic field $B_{\text {crit }}=E_{\text {crit }} / c$ is given by $B_{\text {crit }} \approx 4.4 \times 10^{9} \mathrm{~T}$. It is extremely hard to reach such field strengths in the laboratory, but even stronger fields exist in extra-terrestrial environments, cf. [6-9].

Published by the American Physical Society under the terms of the Creative Commons Attribution 4.0 International license. Further distribution of this work must maintain attribution to the author(s) and the published article's title, journal citation, and DOI. Funded by SCOAP ${ }^{3}$.
As an exception, the Coulomb field of a nucleus with charge $Z q$ exceeds the field strength (1) very close to the nucleus, i.e., on a distance of order $\mathcal{O}(\sqrt{Z} \chi)$. Such a high field strength helps to observe the interaction of electromagnetic fields and the scattering of photons at the nuclear Coulomb field, which can be understood as quantum vacuum refraction (usually referred to as Delbrück scattering [10-12]), has been observed in several experiments, see, e.g., [13-28]. In order to probe the high field in the close vicinity of the nucleus, these photons had an energy of the order of the electron mass or above. As another example, the interaction of the Coulomb fields of two nuclei almost colliding with each other at ultrahigh energies and the resulting emission of a pair of photons has also been observed [29-32].

In contrast, neither the interaction of electromagnetic fields (as predicted by QED) in a regime well below the QED scales $m c^{2}, E_{\text {crit }}$ and $B_{\text {crit }}$, nor quantum vacuum birefringence have been experimentally verified yet. Prominent proposals for ongoing and planned experiments include the interaction of an optical (or near-optical) laser with a (quasi)static magnetic field of a few Tesla, see, e.g., [33-41], the interaction of x-ray free electron laser (XFEL) beams among each other or with optical lasers, see, e.g., [42-47], or the interaction of several optical lasers, see, e.g., [48-58].

Here, we consider a mixed setup where an XFEL beam is scattered at the combination of a strong magnetic field $\mathbf{B}_{\text {ext }}$ superimposed by the Coulomb field $\mathbf{E}_{\text {ext }}$ of a nucleus (as schematically depicted in Fig. 1), see also [59]. This scenario offers several advantages: The nuclear Coulomb 


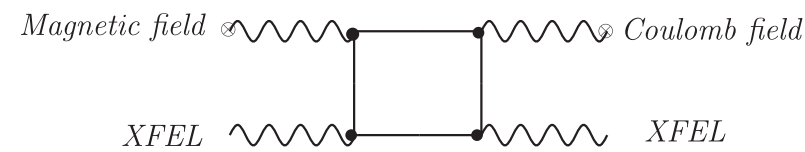

FIG. 1. Exemplary Feynman diagram of the considered process (wavy/straight lines represent photons/electrons).

field facilitates a significant momentum transfer $\Delta \mathbf{k}$ while we still obtain a birefringent signal (where the polarization of the XFEL photon flips). As an important difference to the aforementioned scenarios involving nuclear fields, we obtain a very large interaction volume (which may even contain many nuclei) whose length and timescales are set by the momentum transfer $\Delta \mathbf{k}$ and thus well above the Compton length $\chi$. Hence, all involved field strengths are subcritical, i.e., well below (1).

\section{EULER-HEISENBERG LAGRANGIAN}

Since we are considering slowly varying electric $\mathfrak{E}$ and magnetic $\mathfrak{B}$ fields well below $E_{\text {crit }}$ and $B_{\text {crit }}$, we may start with the lowest-order Euler-Heisenberg Lagrangian $\left(\hbar=c=\epsilon_{0}=\mu_{0}=1\right)$

$$
\mathfrak{Q}=\frac{1}{2}\left(\mathfrak{F}^{2}-\mathfrak{B}^{2}\right)+\xi\left[\left(\mathfrak{F}^{2}-\mathfrak{B}^{2}\right)^{2}+7(\mathfrak{E} \cdot \mathfrak{B})^{2}\right],
$$

with a nonlinearity set by the parameter

$$
\xi=\frac{q^{4}}{360 \pi^{2} m^{4}}=\frac{2 \alpha_{\mathrm{QED}}^{2}}{45 m^{4}}=\frac{\alpha_{\mathrm{QED}}}{90 \pi E_{\mathrm{crit}}^{2}},
$$

where $\alpha_{\mathrm{QED}} \approx 1 / 137$ is the fine-structure constant [2], see also [60-70]. This is a great advantage because we do not have to construct the electron propagator whose explicit form is known in special cases only [71], e.g., in an electromagnetic plane-wave background which facilitates Volkov solutions [72,73], see also [74-77].

In the weak-field limit considered here, we neglect quadratic terms $\mathcal{O}\left(\xi^{2}\right)$. Furthermore, we assume that the magnetic field $\mathbf{B}_{\text {ext }}$ is (approximately) constant. In addition to this magnetic field $\mathbf{B}_{\text {ext }}$ and the static Coulomb field $\mathbf{E}_{\text {ext }}$ of the nucleus, we have the space-time dependent XFEL fields $\mathbf{E}$ and $\mathbf{B}$. Inserting this split $\mathfrak{E}=\mathbf{E}_{\text {ext }}+\mathbf{E}$ and $\mathfrak{B}=$ $\mathbf{B}_{\text {ext }}+\mathbf{B}$ into (2), we obtain the effective Lagrangian for the XFEL fields (cf. [58])

$$
\begin{aligned}
\mathfrak{\Omega}_{\mathrm{XFEL}}= & \frac{1}{2}[\mathbf{E} \cdot(\mathbb{1}+\delta \epsilon) \cdot \mathbf{E}-\mathbf{B} \cdot(\mathbb{1}-\delta \mu) \cdot \mathbf{B}] \\
& +\mathbf{E} \cdot \delta \Psi \cdot \mathbf{B}
\end{aligned}
$$

with the symmetric permittivity/permeability tensors $\delta \epsilon^{i j}=8 \xi E_{\mathrm{ext}}^{i} E_{\mathrm{ext}}^{j}+14 \xi B_{\mathrm{ext}}^{i} B_{\mathrm{ext}}^{j}+4 \xi \delta^{i j}\left(\mathbf{E}_{\mathrm{ext}}^{2}-\mathbf{B}_{\mathrm{ext}}^{2}\right) \quad$ and $\delta \mu^{i j}=8 \xi B_{\mathrm{ext}}^{i} B_{\mathrm{ext}}^{j}+14 \xi E_{\mathrm{ext}}^{i} E_{\mathrm{ext}}^{j}-4 \xi \delta^{i j}\left(\mathbf{E}_{\mathrm{ext}}^{2}-\mathbf{B}_{\mathrm{ext}}^{2}\right)$ plus the symmetry-breaking contribution

$$
\begin{aligned}
\delta \Psi^{i j}= & -8 \xi E_{\mathrm{ext}}^{i} B_{\mathrm{ext}}^{j}+14 \xi B_{\mathrm{ext}}^{i} E_{\mathrm{ext}}^{j} \\
& +14 \xi \delta^{i j}\left(\mathbf{E}_{\mathrm{ext}} \cdot \mathbf{B}_{\mathrm{ext}}\right),
\end{aligned}
$$

which describe the polarizability of the QED vacuum. Note that the latter tensor is not symmetric $\delta \Psi^{i j} \neq \delta \Psi^{j i}$.

The equations of motion stemming from (4) can be cast into the same form as the macroscopic Maxwell equations in a medium $\nabla \cdot \mathbf{D}=0, \nabla \cdot \mathbf{B}=0, \nabla \times \mathbf{E}=-\partial_{t} \mathbf{B}$, and $\nabla \times \mathbf{H}=\partial_{t} \mathbf{D}$, provided that we introduce the electric $\mathbf{D}=(\mathbb{1}+\delta \epsilon) \cdot \mathbf{E}+\delta \Psi \cdot \mathbf{B}$ and magnetic displacement fields $\mathbf{H}=(\mathbb{1}-\delta \mu) \cdot \mathbf{B}-\delta \Psi^{\mathrm{T}} \cdot \mathbf{E}$.

\section{SCATTERING THEORY}

Now we may calculate the scattering of the XFEL beam with standard approaches, see, e.g., [78]. Combining the above Maxwell equations to

$$
\square \mathbf{D}=\nabla \times[\nabla \times(\mathbf{D}-\mathbf{E})]+\partial_{t}[\nabla \times(\mathbf{H}-\mathbf{B})]=\mathbf{J}^{\mathrm{eff}},
$$

where the effective source term $\mathbf{J}^{\text {eff }}$ on the right-hand side is small, allows us to employ the Born approximation. To this end, we split the XFEL field $\mathbf{D}$ into an ingoing plane wave $\mathbf{D}^{\text {in }}$ plus a small scattering contribution $\mathbf{D}^{\text {out }}$ induced by vacuum polarizability $\delta \epsilon, \delta \mu$, and $\delta \Psi$. Assuming a stationary time dependence $e^{-i \omega t}$ for the XFEL field $(\delta \epsilon, \delta \mu$, and $\delta \Psi$ are static), we find

$$
\begin{aligned}
\square \mathbf{D}_{\omega}^{\text {out }}= & -\left(\nabla^{2}+\omega^{2}\right) \mathbf{D}_{\omega}^{\text {out }}=\mathbf{J}_{\omega}^{\mathrm{eff}} \\
= & \nabla \times\left[\nabla \times\left(\delta \epsilon \cdot \mathbf{E}_{\omega}^{\text {in }}+\delta \Psi \cdot \mathbf{B}_{\omega}^{\text {in }}\right)\right] \\
& +i \omega \nabla \times\left(\delta \mu \cdot \mathbf{B}_{\omega}^{\text {in }}+\delta \Psi^{\mathrm{T}} \cdot \mathbf{E}_{\omega}^{\text {in }}\right),
\end{aligned}
$$

where we may approximate $\mathbf{E}_{\omega}^{\text {in }} \approx \mathbf{D}_{\omega}^{\text {in }}$ and $\mathbf{B}_{\omega}^{\text {in }} \approx \mathbf{H}_{\omega}^{\text {in }}$ on the right-hand side within the Born approximation. As usual, solving this Helmholtz differential equation for $\mathbf{D}_{\omega}^{\text {out }}$ with the standard Greens function and considering the behavior at large spatial distances, we find the differential cross section $d \sigma / d \Omega=|\mathfrak{A}|^{2}$ with the scattering amplitude [78]

$$
\mathfrak{A}=\frac{1}{4 \pi\left|\mathbf{D}_{\omega}^{\text {in }}\right|} \mathbf{e}_{\text {out }} \cdot \int d^{3} r \exp \left\{-i \mathbf{k}_{\text {out }} \cdot \mathbf{r}\right\} \mathbf{J}_{\omega}^{\text {eff }},
$$

where $\mathbf{k}_{\text {out }}$ is the wave number and $\mathbf{e}_{\text {out }}$ the polarization unit vector of the scattered (outgoing) XFEL radiation. The effective source term $\mathbf{J}_{\omega}^{\text {eff }}$ contains contributions from $\delta \epsilon$, $\delta \mu$, and $\delta \Psi$ which add up to give the full amplitude

$$
\begin{aligned}
\mathfrak{A}= & \frac{\omega^{2}}{4 \pi} \int d^{3} r \exp \{i \Delta \mathbf{k} \cdot \mathbf{r}\}\left[\mathbf{e}_{\text {out }} \cdot \delta \epsilon \cdot \mathbf{e}_{\text {in }}\right. \\
& +\mathbf{e}_{\text {out }} \cdot \delta \Psi \cdot\left(\mathbf{n}_{\text {in }} \times \mathbf{e}_{\text {in }}\right)+\mathbf{e}_{\text {in }} \cdot \delta \Psi \cdot\left(\mathbf{n}_{\text {out }} \times \mathbf{e}_{\text {out }}\right) \\
& \left.+\left(\mathbf{n}_{\text {out }} \times \mathbf{e}_{\text {out }}\right) \cdot \delta \mu \cdot\left(\mathbf{n}_{\text {in }} \times \mathbf{e}_{\text {in }}\right)\right] .
\end{aligned}
$$


Here $\mathbf{n}_{\text {in }}$ and $\mathbf{n}_{\text {out }}$ are the initial and final propagation directions $\left(\mathbf{k}_{\text {in }}=\omega \mathbf{n}_{\text {in }}\right.$ and $\left.\mathbf{k}_{\text {out }}=\omega \mathbf{n}_{\text {out }}\right)$, respectively, while $\mathbf{e}_{\text {in }}$ and $\mathbf{e}_{\text {out }}$ denote their polarizations. As usual in scattering theory, the oscillating phase is governed by the momentum transfer $\Delta \mathbf{k}=\mathbf{k}_{\text {in }}-\mathbf{k}_{\text {out }}$.

In the following, we only consider the terms stemming from the combined impact of the magnetic field $\mathbf{B}_{\mathrm{ext}}$ and the nuclear Coulomb field $\mathbf{E}_{\text {ext }}$. Keeping only those cross terms, just the symmetry-breaking contribution $\delta \Psi$ in (5) survives, i.e., we focus on the second line of Eq. (9).

Since $\mathbf{B}_{\text {ext }}$ is (nearly) constant, the spatial integral yields the Fourier transform of the nuclear Coulomb field $\mathbf{E}_{\text {ext }}(\mathbf{r})=\mathbf{e}_{r} Q /\left(4 \pi \mathbf{r}^{2}\right)$ with the charge $Q=Z q$ :

$$
\int d^{3} r e^{i \Delta \mathbf{k} \cdot \mathbf{r}} \frac{Q}{4 \pi \mathbf{r}^{2}} \mathbf{e}_{r}=i Q \frac{\Delta \mathbf{k}}{(\Delta \mathbf{k})^{2}} .
$$

An important point here is that the $1 / r^{2}$ scaling from the Coulomb field cancels the $r^{2}$ volume factor in the $d^{3} r$ integration. As a consequence, the spatial integration is cut off by the momentum transfer $\Delta \mathbf{k}$ resulting in a very large interaction volume-which may even span many XFEL wavelengths for small scattering angles $\left|\mathbf{n}_{\text {in }}-\mathbf{n}_{\text {out }}\right| \ll 1$, i.e., in forward direction. Of course, at some point the approximation of a constant $\mathbf{B}_{\text {ext }}$ breaks down.

\section{FORWARD SCATTERING}

The large interaction volume mentioned above goes along with a peak in forward direction, i.e., for small $\Delta \mathbf{k}$, where the signal is enhanced as $\sim 1 /|\Delta \mathbf{k}|$. Since the prefactor is quite small, let us focus on this leading-order contribution $\sim 1 /|\Delta \mathbf{k}|$. Thus, we approximate $\mathbf{n}_{\text {in }} \approx \mathbf{n}_{\text {out }} \rightarrow \mathbf{n}$ in order to simplify the expressions. In this limit, the isotropic contribution $14 \xi \delta^{i j}\left(\mathbf{E}_{\text {ext }} \cdot \mathbf{B}_{\text {ext }}\right)$ in (5) cancels and we are left with the anisotropic terms. For the birefringent signal, where $\mathbf{e}_{\text {in }}$ and $\mathbf{e}_{\text {out }}$ are orthogonal, we may approximate $\mathbf{e}_{\text {out }} \approx \pm \mathbf{n} \times \mathbf{e}_{\text {in }}$ and $\mathbf{e}_{\text {in }} \approx \mp \mathbf{n} \times \mathbf{e}_{\text {out }}$ which simplifies the integrand in (9) to $\pm\left[\mathbf{e}_{\text {out }} \cdot \delta \Psi \cdot \mathbf{e}_{\text {out }}-\mathbf{e}_{\text {in }} \cdot \delta \Psi \cdot \mathbf{e}_{\text {in }}\right]$. Altogether, we get the birefringent amplitude

$$
\begin{aligned}
\mathfrak{A}_{\delta \Psi}^{\perp}= & \pm 6 i \xi \frac{Q}{(\Delta \mathbf{k})^{2}} \frac{\omega^{2}}{4 \pi}\left[\left(\mathbf{e}_{\text {out }} \cdot \mathbf{B}_{\text {ext }}\right)\left(\mathbf{e}_{\text {out }} \cdot \Delta \mathbf{k}\right)\right. \\
& \left.-\left(\mathbf{e}_{\text {in }} \cdot \mathbf{B}_{\text {ext }}\right)\left(\mathbf{e}_{\text {in }} \cdot \Delta \mathbf{k}\right)\right] .
\end{aligned}
$$

Thus, one way to obtain a maximum birefringent signal would be to align the momentum transfer $\Delta \mathbf{k}$ with $\mathbf{B}_{\text {ext }}$ as well as either $\mathbf{e}_{\text {in }}$ or $\mathbf{e}_{\text {out }}$, for example.

In this case, we find the amplitude (up to a sign)

$$
\mathfrak{A}_{\delta \Psi}^{\perp}=6 i \xi B_{\mathrm{ext}} \frac{Q}{|\Delta \mathbf{k}|} \frac{\omega^{2}}{4 \pi}=i \frac{\alpha_{\mathrm{QED}}^{2}}{15 \pi} \frac{q B_{\mathrm{ext}}}{m^{2}} \frac{\omega^{2}}{m^{2}} \frac{Z}{|\Delta \mathbf{k}|} .
$$

Apart from a numerical prefactor, this amplitude scales with the ratios of the optical laser field strength $B_{\text {ext }}$ over the critical field strength $B_{\text {crit }} \approx 4.4 \times 10^{9} \mathrm{~T}$ and the square of the XFEL frequency $\omega$ in comparison to the electron mass $m$.

\section{DETECTABILITY}

Since the amplitude (12) is proportional to $B_{\text {ext }}$ and $\omega^{2}$, it is desirable to have a strong magnetic field and a large XFEL frequency [79]. Ultrahigh field strengths $B_{\text {ext }}=10^{6} \mathrm{~T}$ can be reached in the focus of an ultrastrong optical or near optical laser with an intensity of order $10^{22} \mathrm{~W} / \mathrm{cm}^{2}$ in a colliding-beam setup. Then, inserting an XFEL frequency of $\omega=24 \mathrm{keV}$, which is still within the range of the European XFEL [45,81,82], we obtain an amplitude of around $\mathfrak{A}_{\delta \Psi}^{\perp} \approx 5 \times 10^{-13} Z /|\Delta \mathbf{k}|$ which yields the birefringent differential cross section in forward direction

$$
\frac{d \sigma_{\delta \Psi}^{\perp}}{d \Omega}=\left|\mathfrak{A}_{\delta \Psi}^{\perp}\right|^{2} \sim 10^{-25} \frac{Z^{2}}{(\Delta \mathbf{k})^{2}} .
$$

For a lower frequency of $\omega=1 \mathrm{keV}$, the amplitude would be reduced to $\mathfrak{A}_{\delta \Psi}^{\perp} \approx 10^{-15} Z /|\Delta \mathbf{k}|$ corresponding to a cross section of $10^{-30} Z^{2} /(\Delta \mathbf{k})^{2}$.

The suppression in (13) by more than 20 orders of magnitude is roughly comparable to other proposals for vacuum birefringence experiments, see, e.g., [42,45]. Yet, this suppression does not imply that the effect is beyond reach. In order to demonstrate that, let us discuss two main enhancement factors. The first enhancement factor is a large number $\mathcal{O}\left(10^{11}\right)$ of polarized XFEL photons, analogous to other vacuum birefringence proposals, see, e.g., $[42,45]$. In addition, a large number $N$ of nuclei represents another enhancement factor of our setup. Note that the peak $\propto 1 /(\Delta \mathbf{k})^{2}$ in the forward direction, after integrating over the solid angle $d \Omega$, does only yield a weak (i.e., logarithmic) enhancement. In addition, as explained after (10), this $1 /(\Delta \mathbf{k})^{2}$ behavior is only valid up to minimum values of $|\Delta \mathbf{k}|$ set by the size of the laser focus. For even smaller $|\Delta \mathbf{k}|$, one would have to include the Fourier transform of the dependence of $\mathbf{B}_{\text {ext }}(\mathbf{r})$ or $\mathbf{B}_{\text {ext }}(t, \mathbf{r})$ as an effective form factor.

As one possible scenario (see Fig. 2), one may consider a cubic cluster with an edge length of $100 \mathrm{~nm}$ with typical solid-state density made of carbon, for example. Then, applying a prepulse with a high intensity a bit below $\mathcal{O}\left(10^{22} \mathrm{~W} / \mathrm{cm}^{2}\right)$, one may blow out almost all electrons, leaving behind $N=\mathcal{O}\left(10^{8}\right)$ ionized nuclei. Shortly afterwards, before the Coulomb explosion of the remaining nuclei, one would have them interact with the XFEL superimposed by the main pulse (in the form of a colliding beam setup, for example). To exploit the peak in the forward direction, let us assume a small momentum transfer $\Delta \mathbf{k}$ in the $\mathrm{eV}$ regime (corresponding to scattering angles $\vartheta$ of order millirad). In this case, the amplitudes from the $N=\mathcal{O}\left(10^{8}\right)$ nuclei would have basically the same phase 

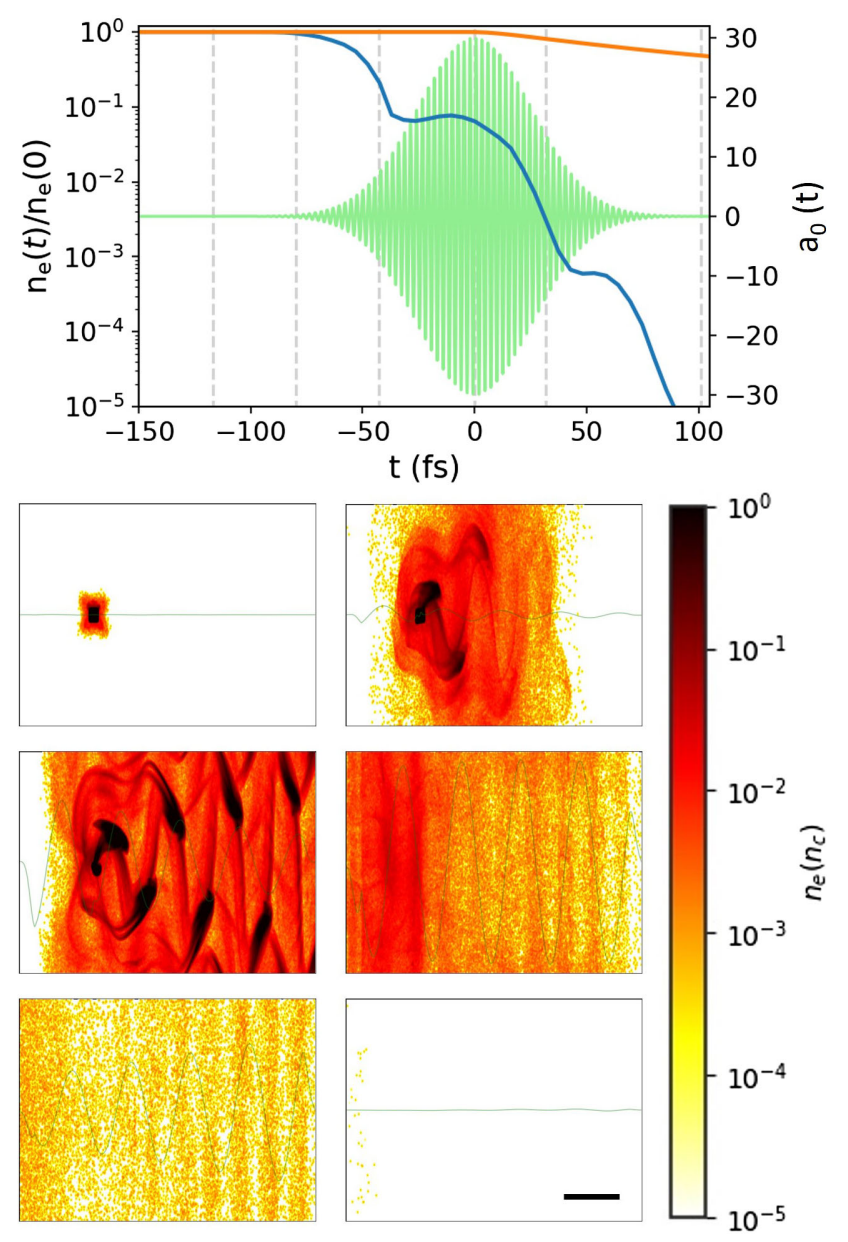

FIG. 2. In an exemplary 3D particle-in-cell (PIC) simulation (PIConGPU), a cubic carbon cluster target with $100 \mathrm{~nm}$ side length and an (initial) electron density $n_{e}$ of $5 \times 10^{22} \mathrm{~cm}^{-3}$ (i.e., $n_{e}=290 n_{c}$ ) was irradiated by a short pulse laser with $800 \mathrm{~nm}$ wavelength, $30 \mathrm{fs}$ width, $1.946 \times 10^{21} \mathrm{~W} / \mathrm{cm}^{2}$ intensity (i.e., $a_{0}=30$ ), incident from the left. In order to assess the number of electrons and ions a transversely oriented XFEL probe beam would observe, we integrated their respective densities perpendicular to the plane shown in the lower panels in a $200 \mathrm{~nm} \times 200 \mathrm{~nm}$ square area. The resulting normalized temporal evolution is plotted in the top: electrons blue, ions orange and the laser electric field is shown in green for reference. The vertical gray dashed lines indicate the times for which the lower panels show the electron density and laser electric field. From top left to bottom right these are $-117,-80,-43,+32$, and +101 fs. We see that the electron number in the interaction volume drops by 5 orders of magnitude while the ion number only decreases moderately (due to the Coulomb explosion). The simulation box has periodic boundaries in the dimensions transverse to the laser; the black scale bar in the bottom right is $500 \mathrm{~nm}$ long.

and thus add up coherently [83], effectively acting as one giant nucleus with charge $Z_{\text {eff }}=N Z$. For much larger $\Delta \mathbf{k}$, one would have to include the spatial distribution of the nuclei in analogy to (10).

For smaller clusters (e.g., $50 \mathrm{~nm}$ ), the electrons would already be blown out before the laser pulse reaches its peak (see the Supplemental Material [80]). Thus, in this second scenario, one could avoid the "prepulse-main pulse" sequence and use one and the same laser pulse for ionization and vacuum polarization. However, in this case (without a colliding beam) one should also take into account the electric field component of the optical laser. This electric field of the laser would then generate additional contributions in $\delta \epsilon$ and $\delta \mu$ after combining it with the nuclear Coulomb field. Inserting those additional contributions in $\delta \epsilon$ and $\delta \mu$ into the full amplitude (9), we find that they exactly cancel the terms from $\delta \Psi$ if the optical laser and the XFEL propagate in the same direction. This cancellation does also occur in the case without the nuclear Coulomb field and demonstrates an important difference between a propagating plane wave (crossed fields) and a pure magnetic field. To avoid this cancellation and obtain a birefringent signal, the XFEL should propagate at a finite angle (e.g., perpendicular) to the propagation direction as well as the magnetic field component of the optical laser. In the perpendicular case, one obtains the same result (12) as for a pure magnetic field, i.e., the electric field of the optical laser does not play a role.

As a third scenario, one could envisage an optical laser focus (expelling the electrons) copropagating with the XFEL pulse through a less dense medium, in analogy to laser wakefield acceleration [84]. In the usual setup, the optical laser would copropagate with the created fully blown-out plasma cavity and thus also with the XFEL. However, this would again lead to the cancellation problem discussed above. To overcome this problem, one could use an optical laser with a propagation direction different from that of the XFEL, whose focus is copropagating with the XFEL pulse.

This can be achieved by laser pulse-front conditioning (which, at the required high intensities, can be accomplished by particular arrangements of optical gratings and cylindrical mirrors [85-88]). Inserting typical numbers such as a density of order $10^{19} \mathrm{~cm}^{-3}$, the number $N$ of ions within the interaction region, i.e., the laser focus with a $2.5 \mu \mathrm{m}$ spot size, traveling over a distance of order millimeter, would be about 2 to 3 orders of magnitude larger $N=\mathcal{O}\left(6 \times 10^{10}\right)$ compared to the first scenario. However, presumably not all of these ions would contribute coherently to the scattering amplitude in view of the larger spatial extent (again compared to the first and second scenario). As an advantage, some of the background processes (see the Supplemental Material [80]) due to the residual electrons and the residual radiation are minimized in the third scenario. In summary, the three scenarios offer different advantages and drawbacks, which should be compared carefully for designing an experimental realization.

Finally, the measurement of the birefringent signal, i.e., the detection of the XFEL photons with flipped polarization could be achieved in complete analogy to other vacuum birefringence proposals, see, e.g., [42,45,89]. 


\section{CONCLUSIONS}

As an example for the QED vacuum nonlinearity, we calculate the scattering of XFEL photons at the combined field of a nucleus plus an external magnetic field (e.g., generated by an optical laser focus), see Fig. 1. In contrast to previous work involving nuclear fields, such as Delbrück scattering, this scenario yields a large interaction volume, which goes along with a peak of the differential cross section in forward direction $d \sigma / d \Omega \sim 1 /(\Delta \mathbf{k})^{2}$.

As another distinction, the scales relevant to our scenario are well below the characteristic scales of QED mentioned in the Introduction, i.e., the critical field strength (1) and the electron mass $m$. Thus, our scenario is within the realm of applicability of the Euler-Heisenberg Lagrangian (2) and the picture of a classical electromagnetic field-especially for the coherent superposition of the signal from many nuclei-instead of the particle (photon) picture often associated with Delbrïck scattering.

In addition to the normal polarization conserving scattering (see the Supplemental Material [80]), we obtain a birefringent signal (12) whose amplitude $\mathfrak{A}_{\delta \Psi}^{\perp}$ is just a little bit smaller.

This vacuum birefringence as a fundamental prediction of QED provides a clear signature of the quantum vacuum polarizability and has not been experimentally verified yet. Together with the anisotropy induced by the magnetic field and the aforementioned peak in forward direction, this birefringence may be used to distinguish the process considered here from other background processes discussed in the Supplemental Material [80].
Using a large number of XFEL photons [81,82] and a large number of nuclei, we show that it might be possible to overcome the suppression of the signal (12) by more than 20 orders of magnitude such that this effect might be observable in the near future at facilities such as the Helmholtz International Beamline for Extreme Fields (HIBEF) at the European XFEL [90]. Since the detection of quantum vacuum birefringence (along the lines of $[42,45])$ has been proposed by the HIBEF consortium as a flagship experiment-where the major requirements of our proposal (including an optical laser focus reaching an intensity of order $10^{22} \mathrm{~W} / \mathrm{cm}^{2}$, temporally matching a polarized XFEL pulse with $10^{11}$ photons, and the highprecision X-ray polarimetry, cf. [89]) largely coincide with those of $[42,45]$ - there is already continuous experimental progress towards reaching these goals.

\section{ACKNOWLEDGMENTS}

We would like to thank C. Schubert as well as R. Sauerbrey, H.-P. Schlenvoigt, R. Shaisultanov, G. Torgrimsson, and other colleagues from the HZDR for helpful discussions. This work was partially funded by the Deutsche Forschungsgemeinschaft (DFG, German Research Foundation)-Project No. 278162697-SFB 1242; as well as by the Center of Advanced Systems Understanding (CASUS) which is financed by Germany's Federal Ministry of Education and Research (BMBF) and by the Saxon Ministry for Science, Culture and Tourism (SMWK) with tax funds on the basis of the budget approved by the Saxon State Parliament.
[1] H. Euler and B. Kockel, Über die Streuung von Licht an Licht nach der Diracschen Theorie, Naturwissenschaften 23, 246 (1935).

[2] W. Heisenberg and H. Euler, Folgerungen aus der Diracschen Theorie des Positrons, Z. Phys. 98, 714 (1936).

[3] R. Karplus and M. Neuman, The scattering of light by light, Phys. Rev. 83, 776 (1951).

[4] F. Sauter, Über das Verhalten eines Elektrons im homogenen elektrischen Feld nach der relativistischen Theorie Diracs, Z. Phys. 69, 742 (1931).

[5] J. Schwinger, On gauge invariance and vacuum polarization, Phys. Rev. 82, 664 (1951).

[6] R. P. Mignani, V. Testa, D. González Caniulef, R. Taverna, R. Turolla, S. Zane, and K. Wu, Evidence for vacuum birefringence from the first optical-polarimetry measurement of the isolated neutron star RX J1856.5-3754, Mon. Not. R. Astron. Soc. 465, 492 (2017).

[7] L. M. Capparelli, A. Damiano, L. Maiani, and A. D. Polosa, A note on polarized light from magnetars, Eur. Phys. J. C 77, 754 (2017).
[8] P. Mészáros, High Energy Radiation from Magnetized Neutron Stars (Chicago University Press, Chicago, 1992).

[9] R. Ruffini, G. Vereshchagin, and S. S. Xue, Electronpositron pairs in physics and astrophysics: From heavy nuclei to black holes, Phys. Rep. 487, 1 (2010).

[10] L. Meitner and H. Kösters, Über die Streuung kurzwelliger $\gamma$-Strahlen, Z. Phys. 84, 137 (1933), with addition from M. Delbrück.

[11] H. A. Bethe and F. Rohrlich, Small angle scattering of light by a Coulomb field, Phys. Rev. 86, 10 (1952).

[12] V. Costantini, B. De Tollis, and G. Pistoni, Nonlinear effects in quantum electrodynamics, Nuovo Cimento Soc. Ital. Fis. 2A, 733 (1971).

[13] G. Jarlskog, L. Jönsson, S. Prünster, H. D. Schulz, H. J. Willutzki, and G. G. Winter, Measurement of Delbrück scattering and observation of photon splitting at high energies, Phys. Rev. D 8, 3813 (1973).

[14] H. Cheng and T. T. Wu, High-Energy Elastic Scattering in Quantum Electrodynamics, Phys. Rev. Lett. 22, 666 (1969). 
[15] H. Cheng and T. T. Wu, High-energy collision processes in quantum electrodynamics. I-IV, Phys. Rev. 182, 1852 (1969); 182, 1868 (1969); 182, 1873 (1969); 182, 1899 (1969).

[16] A. I. Milstein and V. M. Strakhovneko, Quasiclassical approach to the high-energy Delbrück scattering, Phys. Lett. 95A, 135 (1983).

[17] A. I. Milstein and V. M. Strakhovneko, Coherent scattering of high-energy photons in a Coulomb field, Zh. Eksp. Teor. Fiz. 85, 14 (1983); [Sov. Phys. JETP 58, 8 (1983)].

[18] M. Schumacher, I. Borchert, F. Smend, and P. Rullhusen, Delbrück scattering of $2.75 \mathrm{MeV}$ photons by lead, Phys. Lett. 59B, 134 (1975).

[19] H. Falkenberg, A. Hünger, P. Rullhusen, M. Schumacher, A. I. Milstein, and K. Mork, Amplitudes for Delbrück scattering, At. Data Nucl. Data Tables 50, 1 (1992).

[20] H. E. Jackson and K. J. Wetzel, Delbrück Scattering of 10.8-MeV $\gamma$ Rays, Phys. Rev. Lett. 22, 1008 (1969).

[21] R. Moreh and S. Kahana, Delbrück scattering of $7.9 \mathrm{MeV}$ photons, Phys. Lett. 47B, 351 (1973).

[22] S. Kahane, O. Shahal, and R. Moreh, Rayleigh and Delbrück scattering of $6.8-11.4 \mathrm{MeV}$ photons at $\theta=1.5^{\circ}$, Phys. Lett. 66B, 229 (1977).

[23] S. Z. Akhmadaliev et al., Delbrück scattering at energies of 140-450 MeV, Phys. Rev. C 58, 2844 (1998).

[24] A. I. Milstein and M. Schumacher, Present status of Delbrück scattering, Phys. Rep. 243, 183 (1994).

[25] M. Schumacher, Delbrück scattering, Radiat. Phys. Chem. 56, 101 (1999).

[26] P. Papatzacos and K. Mork, Delbrück scattering calculations, Phys. Rev. D 12, 206 (1975).

[27] P. Papatzacos and K. Mork, Delbrück scattering, Phys. Rep. 21, 81 (1975).

[28] P. Rullhusen, W. Mückenheim, F. Smend, M. Schumacher, G. P. A. Berg, K. Mork, and L. Kissel, Test of vacuum polarization by precise investigation of Delbrück scattering, Phys. Rev. C 23, 1375 (1981).

[29] ATLAS Collaboration, Evidence for light-by-light scattering in heavy-ion collisions with the ATLAS detector at the LHC, Nat. Phys. 13, 852 (2017).

[30] ATLAS Collaboration, Observation of Light-by-Light Scattering in Ultraperipheral $\mathrm{Pb}+\mathrm{Pb}$ Collisions with the ATLAS Detector, Phys. Rev. Lett. 123, 052001 (2019).

[31] ATLAS Collaboration, Evidence for light-by-light scattering and searches for axion-like particles in ultraperipheral $\mathrm{PbPb}$ collisions at $\sqrt{s_{N N}}=5.02 \mathrm{TeV}$, Phys. Lett. B 797, 134826 (2019).

[32] D. d'Enterria and G. G. da Silveira, Observing Light-byLight Scattering at the Large Hadron Collider, Phys. Rev. Lett. 111, 080405 (2013); Erratum, Phys. Rev. Lett. 116, 129901 (2016).

[33] D. Valle, A. Ejlli, U. Gastaldi, G. Messineo, Edoardo Milotti, R. Pengo, G.. Ruoso, and G. Zavattini, The PVLAS experiment: Measuring vacuum birefringence and dichroism with a birefringent Fabry Perot cavity, Eur. Phys. J. C 76, 24 (2016).

[34] E. Zavattini, U. Gastaldi, R. Pengo, G. Ruoso, F. D. Valle, and E. Milotti, Measuring the magnetic birefringence of vacuum: The PVLAS experiment, Int. J. Mod. Phys. A 27, 1260017 (2012).
[35] F. D. Valle, G. Di Domenico, U. Gastaldi, E. Milotti, R. Pengo, G. Ruoso, and G. Zavattini, Towards a direct measurement of vacuum magnetic birefringence: PVLAS achievements, Opt. Commun. 283, 4194 (2010).

[36] E. Zavattini et al., New PVLAS results and limits on magnetically induced optical rotation and ellipticity in vacuum, Phys. Rev. D 77, 032006 (2008).

[37] E. Zavattini et al., Experimental Observation of Optical Rotation Generated in Vacuum by a Magnetic Field, Phys. Rev. Lett. 96, 110406 (2006); 99, 129901(E) (2007).

[38] X. Fan et al., The OVAL experiment: A new experiment to measure vacuum magnetic birefringence using high repetition pulsed magnets, Eur. Phys. J. D 71, 308 (2017).

[39] M. T. Hartman, R. Battesti, and C. Rizzo, Characterization of the vacuum birefringence polarimeter at BMV: Dynamical cavity mirror birefringence, IEEE Trans. Instrum. Meas. 68, 2268 (2019).

[40] M. T. Hartman, A. Rivère, R. Battesti, and C. Rizzo, Noise characterization for resonantly-enhanced polarimetric vacuummagnetic-birefringence experiments, Rev. Sci. Instrum. 88, 123114 (2017).

[41] R. Battesti et al., High magnetic fields for fundamental physics, Phys. Rep. 765, 1 (2018).

[42] T. Heinzl, B. Liesfeld, K.-U. Amthor, H. Schwoerer, R. Sauerbrey, and A. Wipf, On the observation of vacuum birefringence, Opt. Commun. 267, 318 (2006).

[43] T. Inada et al., Search for photon-photon elastic scattering in the X-ray region, Phys. Lett. B 732, 356 (2014).

[44] T. Yamaji et al., An experiment of x-ray photon-photon elastic scattering with a Laue-case beam collider, Phys. Lett. B 763, 454 (2016).

[45] H.-P. Schlenvoigt, T. Heinzl, U. Schramm, T. Cowan, and R. Sauerbrey, Detecting vacuum birefringence with $\mathrm{X}$-ray free electron lasers and high-power optical lasers: A feasibility study, Phys. Scr. 91, 023010 (2016).

[46] F. Karbstein and C. Sundqvist, Probing vacuum birefringence using $\mathrm{x}$-ray free electron and optical high-intensity lasers, Phys. Rev. D 94, 013004 (2016).

[47] T. Inada, T. Yamazaki, T. Yamaji, Y. Seino, X. Fan, S. Kamioka, T. Namba, and S. Asai, Probing physics in vacuum using an x-ray free-electron laser, a high-power laser, and a high-field magnet, Appl. Sci. 7, 671 (2017).

[48] A. Di Piazza, A. I. Milstein, and C. H. Keitel, Photon splitting in a laser field, Phys. Rev. A 76, 032103 (2007).

[49] D. Tommasini, A. Ferrando, H. Michinel, and M. Seco, Precision tests of QED and non-standard models by searching photon-photon scattering in vacuum with high power lasers, J. High Energy Phys. 11 (2009) 043.

[50] D. Tommasini and H. Michinel, Light by light diffraction in vacuum, Phys. Rev. A 82, 011803 (2010).

[51] J. K. Koga and T. Hayakawa, Possible Precise Measurement of Delbrück Scattering Using Polarized Photon Beams, Phys. Rev. Lett. 118, 204801 (2017).

[52] B. King and C. Keitel, Photon-photon scattering in collisions of intense laser pulses, New J. Phys. 14, 103002 (2012).

[53] H. Gies, F. Karbstein, and N. Seegert, Quantum reflection as a new signature of quantum vacuum nonlinearity, New J. Phys. 15, 083002 (2013). 
[54] H. Gies, F. Karbstein, and C. Kohlfürst, Photon-photon scattering at the high-intensity frontier, Phys. Rev. D 97, 076002 (2018).

[55] H. Gies, F. Karbstein, and C. Kohlfürst, All-optical signatures of strong-field QED in the vacuum emission picture, Phys. Rev. D 97, 036022 (2018).

[56] B. Döbrich and H. Gies, Interferometry of light propagation in pulsed fields, Europhys. Lett. 87, 21002 (2009).

[57] H. Grote, On the possibility of vacuum QED measurements with gravitational wave detectors, Phys. Rev. D 91, 022002 (2015).

[58] N. Ahmadiniaz, T. E. Cowan, R. Sauerbrey, U. Schramm, H.-P. Schlenvoigt, and R. Schützhold, On the Heisenberg limit for detecting vacuum birefringence, Phys. Rev. D 101, 116019 (2020).

[59] A. Di Piazza and A. I. Milstein, Delbrück scattering in combined Coulomb and laser fields, Phys. Rev. A 77, 042102 (2008).

[60] F. Karbstein, Probing vacuum polarization effects with highintensity lasers, Particles 3, 39 (2020).

[61] B. King and T. Heinzl, Measuring vacuum polarisation with high power lasers, High Power Laser Sci. Eng. 4, E5 (2016).

[62] F. Karbstein, The quantum vacuum in electromagnetic fields: From the Heisenberg-Euler effective action to vacuum birefringence, in Proceedings of the Helmholtz International Summer School 2016 (HQ 2016), Dubna, Russia, 2016 (Verlag Deutsches Elektronen-Synchrotron, DESY-PROC, Hamburg, 2017), pp. 44-57.

[63] A. Di Piazza, C. Müller, K. Z. Hatsagortsyan, and C. H. Keitel, Extremely high-intensity laser interactions with fundamental quantum systems, Rev. Mod. Phys. 84, 1177 (2012).

[64] G. V. Dunne, The Heisenberg-Euler effective action: 75 years on, Int. J. Mod. Phys. A 27, 1260004 (2012).

[65] S.Z. Akhmadaliev et al., Experimental Investigation of High-Energy Photon Splitting in Atomic Fields, Phys. Rev. Lett. 89, 061802 (2002).

[66] Z. Bialynicka-Birula and I. Bialynicki-Birula, Nonlinear effects in quantum electrodynamics. Photon propagation and photon splitting in an external field, Phys. Rev. D 2, 2341 (1970).

[67] S. L. Adler, J. N. Bahcall, C. G. Callan, and M. N. Rosenbluth, Photon splitting in a strong magnetic field, Phys. Rev. Lett. 25, 1061 (1970).

[68] S. L. Adler, Photon splitting and photon dispersion in a strong magnetic field, Ann. Phys. (N.Y.) 67, 599 (1971).

[69] S. L. Adler and C. Schubert, Photon Splitting in a Strong Magnetic Field: Recalculation and Comparison with Previous Calculations, Phys. Rev. Lett. 77, 1695 (1996).

[70] J. S. Toll, The dispersion relation for light and its application to problems involving electron pairs, Ph.D. thesis, Princeton University, Princeton, NJ, 1952 (unpublished).

[71] One could derive the same result from QED Feynman diagrams such as in Fig. 1, see also the Supplemental Material. Since three vertices (the two XFEL photons and the external magnetic field) correspond to low energies and momenta (i.e., well below the electron mass $m$ ), energymomentum conservation implies that the fourth vertex (representing the Coulomb field) does also involve low energies and momenta. In fact, for the case of forward scattering considered here, these energies and momenta are extremely low. Thus, we may use the low-energy limit of the QED Feynman diagrams, which are equivalent to the EulerHeisenberg Lagrangian (2). Note that the situation is different for Delbrück scattering where only two vertices (the two XFEL photons) correspond to low energies and momenta such that the other two (representing the Coulomb field) can involve high momenta.

[72] D. M. Volkov, Über eine Klasse von Lösungen der Diracschen Gleichung, Z. Phys. 94, 250 (1935).

[73] P. J. Redmond, Solution of the Klein-Gordon and Dirac equations for a particle with a plane electromagnetic wave and a parallel magnetic field, J. Math. Phys. (N.Y.) 6, 1163 (1965).

[74] A. I. Milstein, I. S. Terekhov, U. D. Jentschura, and C. H. Keitel, Laser-dressed vacuum polarization in a Coulomb field, Phys. Rev. A 72, 052104 (2005).

[75] A. Di Piazza, K. Z. Hatsagortsyan, and C. H. Keitel, Nonperturbative Vacuum-Polarization Effects in Proton-Laser Collisison, Phys. Rev. Lett. 100, 010403 (2008).

[76] A. Di Piazza and A. I. Milstein, Quasiclassical approach to high-energy QED processes in strong laser and atomic fields, Phys. Lett. B 717, 224 (2012).

[77] A. Di Piazza and A. I. Milstein, Ultrarelativistic quasiclassical wave functions in strong laser and atomic fields, Phys. Rev. A 89, 062114 (2014).

[78] J. D. Jackson, Classical Electrodynamics (John Wiley \& Sons, New York, 2007).

[79] The case of smaller frequencies is discussed in the Supplemental Material [80].

[80] See Supplemental Material at http://link.aps.org/ supplemental/10.1103/PhysRevD.104.L011902, for further details and a discussion of potential background processes.

[81] T. Tschentscher, C. Bressler, J. Grünert, A. Madsen, A. Mancuso, M. Meyer, A. Scherz, H. Sinn, and U. Zastrau, Photon beam transport and scientific instruments at the European XFEL, Appl. Sci. 7, 592 (2017).

[82] https://www.xfel.eu.

[83] Because the magnetic field $\mathbf{B}_{\text {ext }}$ is fixed externally (and approximately constant), the $\delta \Psi$ contributions from the nuclei would just add up (in the same way as their Coulomb fields $\mathbf{E}_{\text {ext }}$ add up) and thus the total amplitude $\mathfrak{A}_{\delta \Psi}$ is simply the sum of the amplitudes from all the nuclei separately. Note that the recoil of the heavy nuclei is negligible due to the small momentum transfer (in the eV regime) and the tight spatial confinement of the nuclear wave functions (far below $100 \mathrm{~nm}$ ), leading to a momentum uncertainty much larger than the recoil.

[84] E. Esarey, C. B. Schroeder, and W. P. Leemans, Physics of laser-driven plasma-based electron accelerators, Rev. Mod. Phys. 81, 1229 (2009).

[85] A. Debus, M. Bussmann, M. Siebold, A. Jochmann, U. Schramm, T. E. Cowan, and R. Sauerbrey, Traveling-wave Thomson scattering and optical undulators for high-yield EUV and x-ray sources, Appl. Phys. B 100, 61 (2010).

[86] K. Steiniger, M. Bussmann, R. Pausch, T. Cowan, A. Irman, A. Jochmann, R. Sauerbrey, U. Schramm, and A. Debus, Optical free-electron lasers with traveling-wave Thomson scattering, J. Phys. B 47, 234011 (2014). 
[87] A. Debus, R. Pausch, A. Huebl, K. Steiniger, R. Widera, T. E. Cowan, U. Schramm, and M. Bussmann, Circumventing the Dephasing and Depletion Limits of Laser-Wakefield Acceleration, Phys. Rev. X 9, 031044 (2019).

[88] K. Steiniger, D. Albach, M. Bussmann, M. Loeser, R. Pausch, F. Röser, U. Schramm, M. Siebold, and A. Debus,
Building an optical free-electron laser in the travelingwave Thomson-scattering geometry, Front. Phys. 6, 155 (2019).

[89] K. S. Schulze, Fundamental limitations of the polarization purity of x rays, APL Photonics 3, 126106 (2018).

[90] http://www.hibef.eu. 